\title{
Checklist of the agaricoid and similar morphology mycobiota of Paraíba State, Brazil
}

\author{
Juliane de Castro Valões-Araújo ${ }^{1}$, and (Delipe Wartchow ${ }^{2}$
}

How to cite: Valões-Araújo, J.C. \& Wartchow F. 2021. Checklist of the agaricoid and similar morphology mycobiota of Paraíba State, Brazil. Hoehnea 48: e1322020. https://doi.org/10.1590/2236-8906-132/2020

\begin{abstract}
Checklist of the agaric mycobiota and similar morphology of Paraíba State, Brazil). Brazil has a rich biodiversity, but many species remain to be discovered, studied and cataloged. In the Paraíba State, taxonomy studies on agaric fungi are still infrequent. Thus, this present work carried out a bibliographic review of fungal species belonging to orders Agaricales, Boletales, Cantharellales, Gomphales and Russulales from the Paraíba State previously described or mentioned, because the knowledge about the fungal diversity in the region still remain disaggregated and poorly known. Thus, we organize and present information about the geographic distribution of the taxa in this checklist format. For the compilation of the results, for each species we present the published herbarium numbers and their respective citations. As results, we found 79 taxa belonging 41 genera, 22 families and five orders occurring in areas of the Atlantic Forest and Caatinga. So, this review is extremely important for the dissemination of the valuation of the State's biodiversity providing information about our Brazilian biomes. Keywords: Agaricomycetes, basidiomycota, neotropics, voucher
\end{abstract}

RESUMO - (Checklist da micota agaricoide e de morfologia similar do Estado da Paraíba, Brasil). O Brasil possui uma rica biodiversidade, por isso existem muitas espécies a serem descobertas, estudadas e catalogadas. No Estado da Paraíba, ainda são poucos os estudos envolvendo taxonomia. Com isso, o trabalho realizou um levantamento bibliográfico das espécies fúngicas do Estado da Paraíba já descritas ou citadas, pois o conhecimento sobre a diversidade de fungos que possuímos ainda é muito desagregado e pouco conhecido. Dessa forma, organizamos e apresentamos informações sobre a distribuição geográfica dos táxons em formato de check list. Para a compilação dos resultados, para cada espécie apresentamos os números de tombamento do herbário publicados e suas citações. Como resultado, listamos 79 táxons pertencendo a 41 gêneros, 22 famílias e cinco ordens ocorrendo em áreas de Mata Atlântica e Caatinga. Sendo assim, este trabalho é de extrema importância para a avaliação da biodiversidade do Estado providenciando informação acerca os biomas brasileiros. Palavras-chave: Agaricomycetes, basidiomycota, neotrópicos, voucher

\section{Introduction}

It is known that Brazil has a rich biodiversity, with Cerrado and Mata Atlântica biomes considered as hotspots for biodiversity conservation, and the Pantanal biome as the largest swamped area of the world (Brandon et al. 2005). The recognition of the tropical fungal biodiversity, on which many taxa remain undescribed and also in risk of extinction, is very important but, due of their magnitude, too many species remain to be discovered basing in taxonomic, systematic, ecological, morphological, studies on biological conservation and biodiversity as well palaeobiology, improving the knowledge about these organisms (Hawksworth 1991, Funk et al. 2002).

Among Basidiomycota the fungi with agaricoid habit, formally called mushrooms, were formerly grouped in 16 families among the suborders Agaricineae, Boletineae and Russulineae, according to most known classification of the order Agaricales (Singer 1986). During phylogenetic systematic studies, Matheny et al. (2006) divided the euagarics in six clades:
1) Plicaturopsidoid; 2) Pluteoid encompassing the families Pluteaceae (Pluteus Fr. and Volvariella Speg. ), Amanitaceae (Amanita Pers. and Limacella Earle ) and Hohenbuehelia Schulzer; 3) Hygrophoroid with Hygrophoraceae and some members previously belonging to Tricholomataceae; 4) Marasmioid, with Marasmiaceae, Omphalotaceae, Cyphellaceae, Physalacriaceae and Schizophyllaceae; 5) Tricholomatoide, with members Tricholomataceae sensu stricto, Lyophyllaceae, Entolomataceae and Mycenaceae; and 6) Agaricoid including Cystodermataceae, Nidulariaceae, Agaricaceae, Inocybaceae, Bolbitiaceae, Cortinariaceae, Psathyrellaceae, Crepidotaceae among other.

Recent studies by Dentinger et al. (2016) described Agaricales divided into the suborders Hygrophorineae (containing Clavariaceae and Hygrophoraceae), Pleurotineae (Pleurotaceae and Pterulaceae), Schizophyllineae (Fistulinaceae and Schizophyllaceae), Marasmiineae (Cyphellaceae, Mararmiaceae, Mycenaceae, Omphalotaceae and Physalacriaceae), Tricholomatineae (Entolomataceae, Lyophyllaceae, Macrocystidiaceae and Tricholomataceae),

1. Universidade Federal do Rio Grande do Norte, Programa de Pós-Graduação em Sistemática e Evolução, Avenida Senador Salgado Filho, 3000, Campus Universitário, Lagoa Nova, 59064-741 Natal, RN, Brazil

2. Universidade Federal da Paraíba, Departamento de Sistemática e Ecologia, Cidade Universitária s/n, Campus I, Castelo Branco, 58051-900 João Pessoa, PB, Brazil

3. Corresponding author: fwartchow@yahoo.com.br 
Pluteineae (Amanitaceae and Pluteaceae), and Agaricineae (Agaricaceae, Psathyrellaceae, Strophariaceae, Crepidotaceae, Hymenogastraceae and Inocybaceae). The families previously considered in Agaricales, as Boletaceae (habit boletoid) and Russulaceae (russuloid), were separated in the orders Boletales e Russulales (Binder \& Hibbett 2006, Miller et al. 2006).

Taxonomic studies on agaric fungi in Paraíba State were infrequent in the last century, with species described or cited by Singer (1961, 1965, 1966, 1973a, 1973b, 1976, 1977), Horak (1977) and Oliveira \& Sousa (1995, 1996, 2002). Only recently the studies in systematic and taxonomy of agaricoid fungi in the state were intensified with Wartchow et al. (2012, 2015, 2017), Magnago et al. (2013, 2015, 2017 , 2019), Pinheiro \& Wartchow (2013), Pinheiro et al. (2013), Gomes \& Wartchow (2014), Henkel et al. (2014), Coimbra et al. (2015), Silva-Junior \& Wartchow (2015), Sá \& Wartchow (2016), Barbosa-Silva et al. (2017, 2020), Nascimento \& Wartchow (2018), Silva-Filho et al. (2019, 2020, 2021), Barbosa-Silva \& Wartchow (2020) and Sulzbacher et al. (2020). They described or reported more taxa for the region.

The objective of this work is to perform a bibliographic overview of agaric fungi of Paraíba State, for improve the current status of knowledge of this group of fungi in this region. This knowledge is essential for instruct and raise awareness about the importance of the biological conservation in ecosystems and the self-sustainability (Lodge et al. 1995, Straatsma et al. 2001).

\section{Materials \& Methods}

The Paraíba State (figure 1) is localized at the Northeastern region of Brazil and occupies an area of $56,372 \mathrm{~km}^{2}$, corresponding to less than $1 \%$ of the national territory (Francisco et al. 2015). The geographical relief is diversified, with parts with humid, subhumid and semiarid climates.
Regarding to vegetation, the state has forests formation as restinga, mangrove, ombrophilous and semideciduous forests, humid forests, tabuleiros costeiros, arbustive-arborous caatinga, open arbustive caatinga and other (Governo do Estado Paraíba 2006). The names of the biomes are according to IBGE (2004).

For the elaboration of the map, we use Google Earth ${ }^{\mathrm{TM}}$. For the species compilation we performed a bibliographic survey in which the species are presented with their citation and region of the State where the materials were collected, including respective herbarium voucher's deposit. When the authors did not inform herbarium number, we consulted the INCT-Virtual Herbarium of Flora and Fungi website (https://specieslink.net/). If we do not find the herbarium code and number, we inform the collection number and the herbarium acronym where supposedly the material is deposited [e.g., Singer B 3341 (BAFC, LIL), M.A. Neves 412 (FLOR), A.N.M. Furtado 32 (FLOR) and other]. Taxonomic position of the generic names is based in $\mathrm{He}$ et al. (2019), Kalichman et al. (2020) and Wijayawardene et al. (2020). Epithets and species authority are based in Mycobank (Crous et al. 2004, Robert et al. 2113). Herbaria codes follow Thiers (continuously updated), except for UFRN-Fungos (Departamento de Botânica e Zoologia, Universidade Federal do Rio Grande do Norte).

\section{Results and discussion}

In this study we are considering only taxa with agaricoid and also similar habit as boletoid, russuloid, cantharelloid (Cantharellales, Moncalvo et al. 2006) and gomphoid (Gomphales, Hosaka et al. 2006).

After survey of 30 works (including article, books and books chapters) we found 79 taxa belonging to 41 genera, 22 families and five orders (figure 2, table 1), collected from eight areas belonging six municipalities:
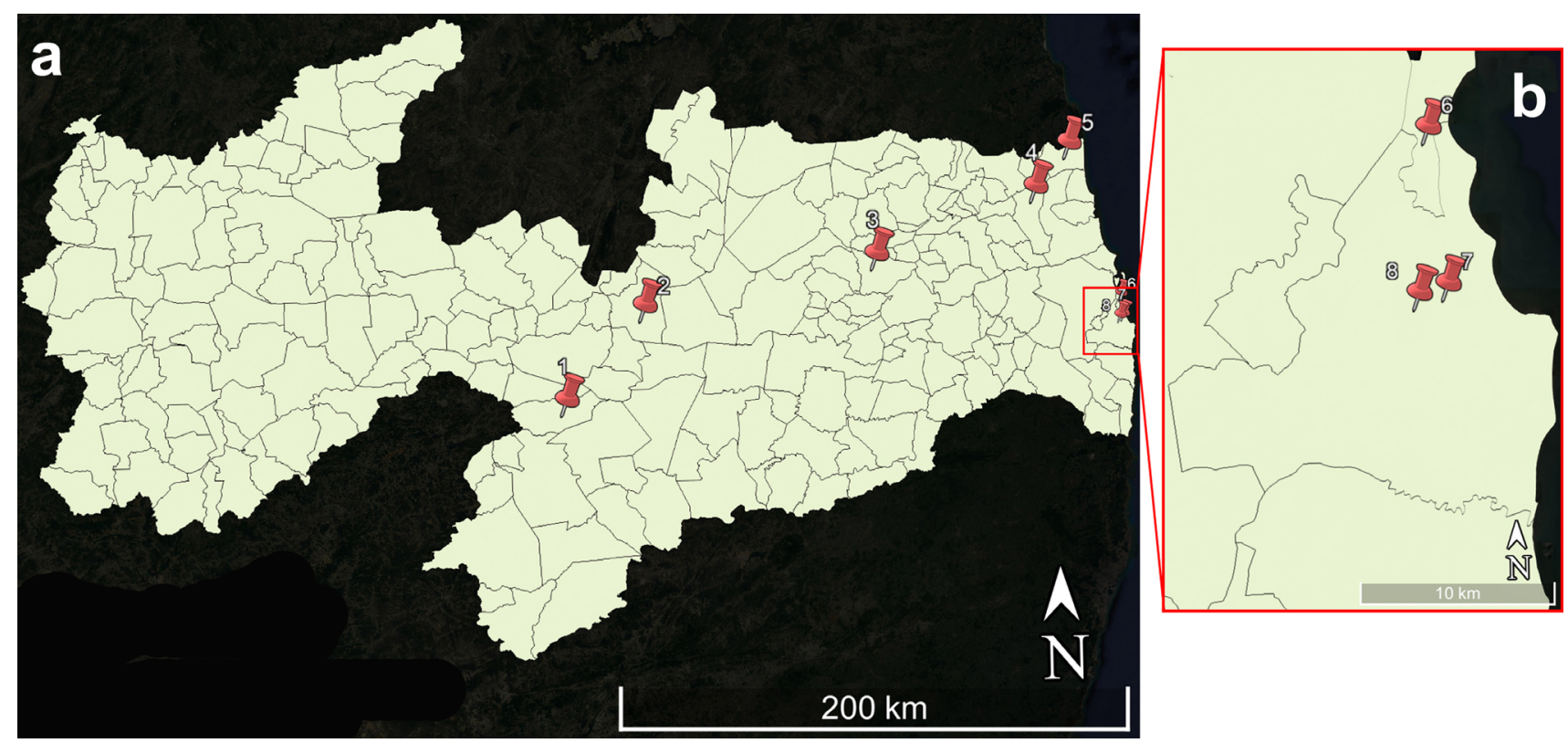

Figure 1. Collection sites of agaricoid fungi in the Paraíba State, Brazil. a. Map with its respective municipalities. 1. São José dos Cordeiros. 2. Juazeirinho. 3. Areia. 4. Mamanguape. 5. Mataraca. 6. Cabedelo. b. Magnification of collection sites in the city of João Pessoa. 7. Jardim Botânico Benjamin Maranhão. 8. Universidade Federal da Paraíba. Source: modified fom Google Earth. 


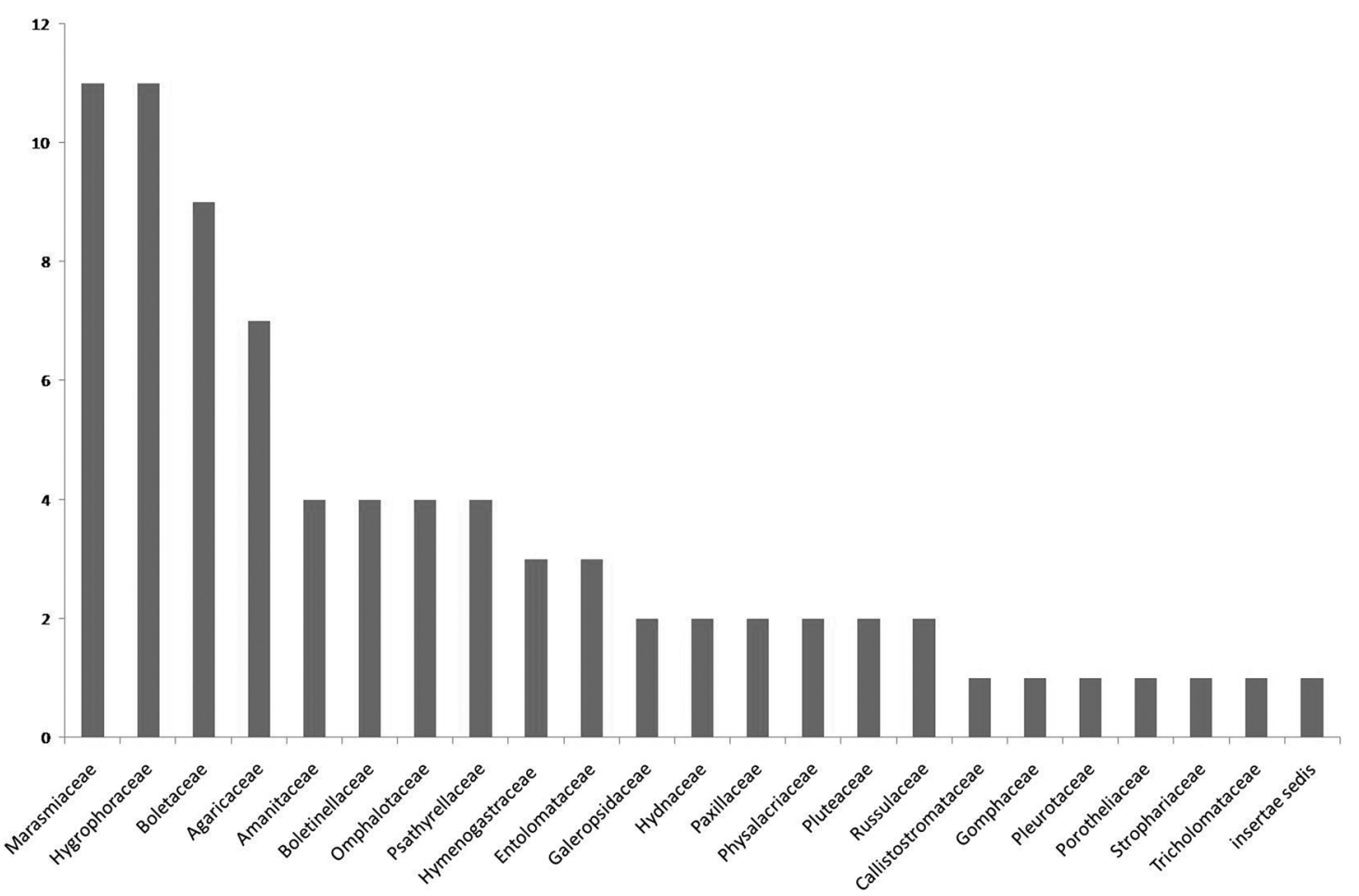

Figure 2. Graphic showing the number of taxa of agaricoid fungi per family recorded from the Paraíba State, Brazil.

Table 1. Checklist of agaricoid and similar morphology fungi from the State of Paraíba, Brazil and their respective localities, authorities and references.

\begin{tabular}{|c|c|c|c|}
\hline Order / Family / Taxon's name & Voucher, Herbaria or Collector number & Locality & Reference \\
\hline $\begin{array}{l}\text { Agaricales } \\
\text { Agaricaceae } \\
\text { Agaricus moelleri Wasser }\end{array}$ & JPB 46270 & $\begin{array}{l}\text { São José dos Cordeiros, } \\
\text { RPPN Fazenda Almas }\end{array}$ & Magnago et al. (2013) \\
\hline $\begin{array}{l}\text { Chlorophyllum molybdites } \\
\text { (G. Mey.) Massee }\end{array}$ & JPB 47307 & $\begin{array}{l}\text { Juazeirinho, Fazenda } \\
\text { Unha-de-Gato }\end{array}$ & Magnago et al. (2013) \\
\hline Coprinus calyptratus Peck & JPB 46272 & $\begin{array}{l}\text { São José dos Cordeiros, } \\
\text { RPPN Fazenda Almas }\end{array}$ & $\begin{array}{c}\text { Magnago et al. (2013 as } \\
\text { 'Coprinus xerophilus Bogart'), } \\
\text { Gomes \& Wartchow (2018) }\end{array}$ \\
\hline $\begin{array}{l}\text { Lepiota erythrosticta } \\
\text { (Berk. \& Broome) Sacc. }\end{array}$ & A.N.M. Furtado 32 (FLOR) & $\begin{array}{l}\text { São José dos Cordeiros, } \\
\text { RPPN Fazenda Almas }\end{array}$ & $\begin{array}{l}\text { Magnago et al. }(2013 \text { as } \\
\text { 'Lepiota erythosticta') }\end{array}$ \\
\hline $\begin{array}{l}\text { Leucoagaricus americanus } \\
\text { (Peck) Vellinga }\end{array}$ & JPB 46277 & $\begin{array}{l}\text { São José dos Cordeiros, } \\
\text { RPPN Fazenda Almas }\end{array}$ & Magnago et al. (2013) \\
\hline $\begin{array}{l}\text { Leucocoprinus birnbaumii } \\
\text { (Corda) Singer }\end{array}$ & M.A. Neves 412 (FLOR) & $\begin{array}{l}\text { Juazeirinho, Fazenda Unha } \\
\text { de Gato; São José dos } \\
\text { Cordeiros, RPPN Fazenda }\end{array}$ & Magnago et al. (2013) \\
\hline $\begin{array}{l}\text { Leucocoprinus fragilissimus } \\
\text { (Berk \&. M.A. Curtis) Pat. }\end{array}$ & JPB 50674 & $\begin{array}{l}\text { João Pessoa, Jardim } \\
\text { Botânico Bejamin } \\
\text { Maranhão }\end{array}$ & Magnago et al. (2015) \\
\hline $\begin{array}{l}\text { Amanitaceae } \\
\qquad \text { Amanita crebresulcata } \text { Bas }\end{array}$ & JPB 50676 & $\begin{array}{c}\text { João Pessoa, Campus I } \\
\text { UFPB }\end{array}$ & Magnago et al. (2015) \\
\hline $\begin{array}{l}\text { Amanita viscidolutea Menolli, } \\
\text { Capelari \& Baseia }\end{array}$ & URM 82095 & $\begin{array}{l}\text { Mataraca, Millenium } \\
\text { Inorganic Chemicals } \\
\text { Minning a Crystal } \\
\text { Company }\end{array}$ & Wartchow et al. (2012) \\
\hline $\begin{array}{l}\text { Limacella brunneovenosa C.C. } \\
\text { Nacimento \& Wartchow }\end{array}$ & JPB 62772 & $\begin{array}{c}\text { Cabedelo, FLONA } \\
\text { Restinga de Cabedelo }\end{array}$ & Nacimento \& Wartchow (2018) \\
\hline Limacella $\mathrm{sp}$. & JPB 47308 & $\begin{array}{l}\text { Juazeirinho, Fazenda } \\
\text { Unha de Gato }\end{array}$ & Magnago et al. (2013) \\
\hline
\end{tabular}




\begin{tabular}{|c|c|c|c|}
\hline \multicolumn{4}{|l|}{ Table 1 (continued) } \\
\hline Order / Family / Taxon's name & Voucher, Herbaria or Collector number & Locality & Reference \\
\hline \multicolumn{4}{|l|}{ Callistostromataceae } \\
\hline $\begin{array}{l}\text { Macrocybe praegrandis } \\
\text { (Berk.) Pegler \& Lodge }\end{array}$ & Singer B 3341 (BAFC, LIL) & João Pessoa & $\begin{array}{l}\text { Singer [1966, as Tricholoma } \\
\text { praegrande (Berk.) Sacc.] }\end{array}$ \\
\hline \multicolumn{4}{|l|}{ Entolomataceae } \\
\hline $\begin{array}{c}\text { Entoloma bloxamii } \\
\text { (Berk. \& Broome) Sacc. }\end{array}$ & JPB 50671 & $\begin{array}{c}\text { Joao Pessoa, Campus I } \\
\text { UFPB }\end{array}$ & Magnago et al. (2015) \\
\hline $\begin{array}{l}\text { Entoloma cantharelluloides } \\
\text { (Singer) E. Horak }\end{array}$ & Singer B 3360 (BAFC) & João Pessoa & $\begin{array}{c}\text { Singer (1965 as Rhodophyllus), } \\
\text { Horak (1977) }\end{array}$ \\
\hline $\begin{array}{l}\text { Rhodocybe crepidotoides } \\
\text { Singer }\end{array}$ & Singer B 3333 (BAFC) & João Pessoa & Singer (1973a) \\
\hline $\begin{array}{l}\text { Galeropsidaceae } \\
\text { Panaeolus antillarum (Fr.) } \\
\text { Dennis }\end{array}$ & JPB 43133 & $\begin{array}{l}\text { São José dos Cordeiros, } \\
\text { RPPN Fazenda Almas }\end{array}$ & Magnago et al. (2013) \\
\hline $\begin{array}{l}\text { Panaeolus papilionaceus } \\
\text { Ew. Gerhardt }\end{array}$ & M.A. Neves 421 (FLOR) & $\begin{array}{l}\text { São José dos Cordeiros, } \\
\text { RPPN Fazenda Almas }\end{array}$ & Magnago et al. (2013) \\
\hline \multicolumn{4}{|l|}{ Hygrophoraceae } \\
\hline $\begin{array}{l}\text { Camarophyllus paraiboensis } \\
\text { Singer }\end{array}$ & Singer B 3334 (BAFC) & João Pessoa & Singer (1977) \\
\hline $\begin{array}{l}\text { Cuphophyllus buccinulus } \\
\text { (Speg.) Courtec. }\end{array}$ & A.C. Magnago 27 (FLOR) & $\begin{array}{l}\text { São José dos Cordeiros, } \\
\text { RPPN Fazenda Almas }\end{array}$ & $\begin{array}{l}\text { Magnago et al. [2013 as } \\
\text { 'Camarophyllus buccinulus } \\
\text { (Speg.) Pegler'] }\end{array}$ \\
\hline $\begin{array}{l}\text { Hygrocybe aurantiomagnifica } \\
\text { Silva-Filho \& Wartchow }\end{array}$ & JPB 62773 & $\begin{array}{l}\text { Areia, Parque Estadual } \\
\text { Mata do Pau-Ferro }\end{array}$ & Silva-Filho et al. (2019) \\
\hline Hygrocybe batistae Singer & JPB 44297 & $\begin{array}{l}\text { Mamanguape, REBio } \\
\text { Guaribas }\end{array}$ & Magnago et al. (2015) \\
\hline $\begin{array}{l}\text { Hygrocybe hypohaemacta } \\
\text { (Corner) Pegler }\end{array}$ & M.A. Neves 413 (FLOR) & $\begin{array}{l}\text { São José dos Cordeiros, } \\
\text { RPPN Fazenda Almas }\end{array}$ & Magnago et al. (2013) \\
\hline $\begin{array}{l}\text { Hygrocybe occidentalis var. } \\
\text { scarletina Pegler }\end{array}$ & JPB 44272, JPB 44273, JPB 44274 & $\begin{array}{l}\text { Mamanguape, REBio } \\
\text { Guaribas }\end{array}$ & Magnago et al. (2015) \\
\hline Hygrocybe paraibensis Singer & Singer B 3320 (LIL) & João Pessoa & Singer (1965) \\
\hline Hygrocybe rhodoleuca Singer & Singer B 3345 (BAFC, LIL) & João Pessoa & Singer (1973a) \\
\hline $\begin{array}{l}\text { Hygrocybe subcaespitosa } \\
\text { (Murrill) Lodge \& Pegler }\end{array}$ & $\begin{array}{c}\text { JPB } 44265, \text { JPB } 44266, \text { JPB } 44271, \\
\text { JPB } 44275, \text { JPB } 44279, \text { JPB } 44280, \\
\text { JPB } 44283\end{array}$ & $\begin{array}{l}\text { Mamanguape, REBio } \\
\text { Guaribas }\end{array}$ & Magnago et al. (2015) \\
\hline $\begin{array}{l}\text { Hygrocybe trinitensis (Dennis) } \\
\text { Pegler }\end{array}$ & JPB 44285 & $\begin{array}{l}\text { Mamanguape, REBio } \\
\text { Guaribas }\end{array}$ & Magnago et al. (2015) \\
\hline Hygrotrama leucopus Singer & Singer B 3344 (BAFC) & João Pessoa & Singer (1973a) \\
\hline \multicolumn{4}{|l|}{ Hymenogastraceae } \\
\hline $\begin{array}{l}\text { Gymnopilus } \\
\text { purpureogramminicola Silva- } \\
\text { Junior \& Wartchow }\end{array}$ & JPB 58545 & $\begin{array}{c}\text { João Pessoa, UFPB } \\
\text { Campus I }\end{array}$ & $\begin{array}{l}\text { Silva-Junior \& Wartchow } \\
\qquad(2015)\end{array}$ \\
\hline $\begin{array}{l}\text { Gymnopilus } \\
\text { purpureosquamulosus Høil. }\end{array}$ & JPB 46297 & $\begin{array}{l}\text { São José dos Cordeiros, } \\
\text { RPPN Fazenda Almas }\end{array}$ & $\begin{array}{c}\text { Magnago et al. [2013, as 'G. } \\
\text { purpureosquamulosus (Peck) } \\
\text { Singer' sic.] }\end{array}$ \\
\hline Gymnopilus subtropicus Hesler & JPB 50672 & $\begin{array}{l}\text { João Pessoa, Jardim } \\
\text { Botânico Bejamin } \\
\text { Maranhão }\end{array}$ & Magnago et al. (2015) \\
\hline \multicolumn{4}{|l|}{ Marasmiaceae } \\
\hline $\begin{array}{l}\text { Crinipellis sapindacearum } \\
\text { Singer }\end{array}$ & Singer 3377 (F 3640) & João Pessoa & Singer (1976) \\
\hline $\begin{array}{l}\text { Marasmius crinis-equi F. } \\
\text { Muell. ex Kalchbr. }\end{array}$ & $\begin{array}{l}\text { JPB } 48125, \text { JPB 48129, JPB 48149, } \\
\text { JPB 48186, JPB 48163, JPB } 48125\end{array}$ & $\begin{array}{l}\text { João Pessoa, UFPB } \\
\text { Campus I, Jardim } \\
\text { Botânico Bejamin } \\
\text { Maranhão }\end{array}$ & Magnago et al. (2015) \\
\hline
\end{tabular}




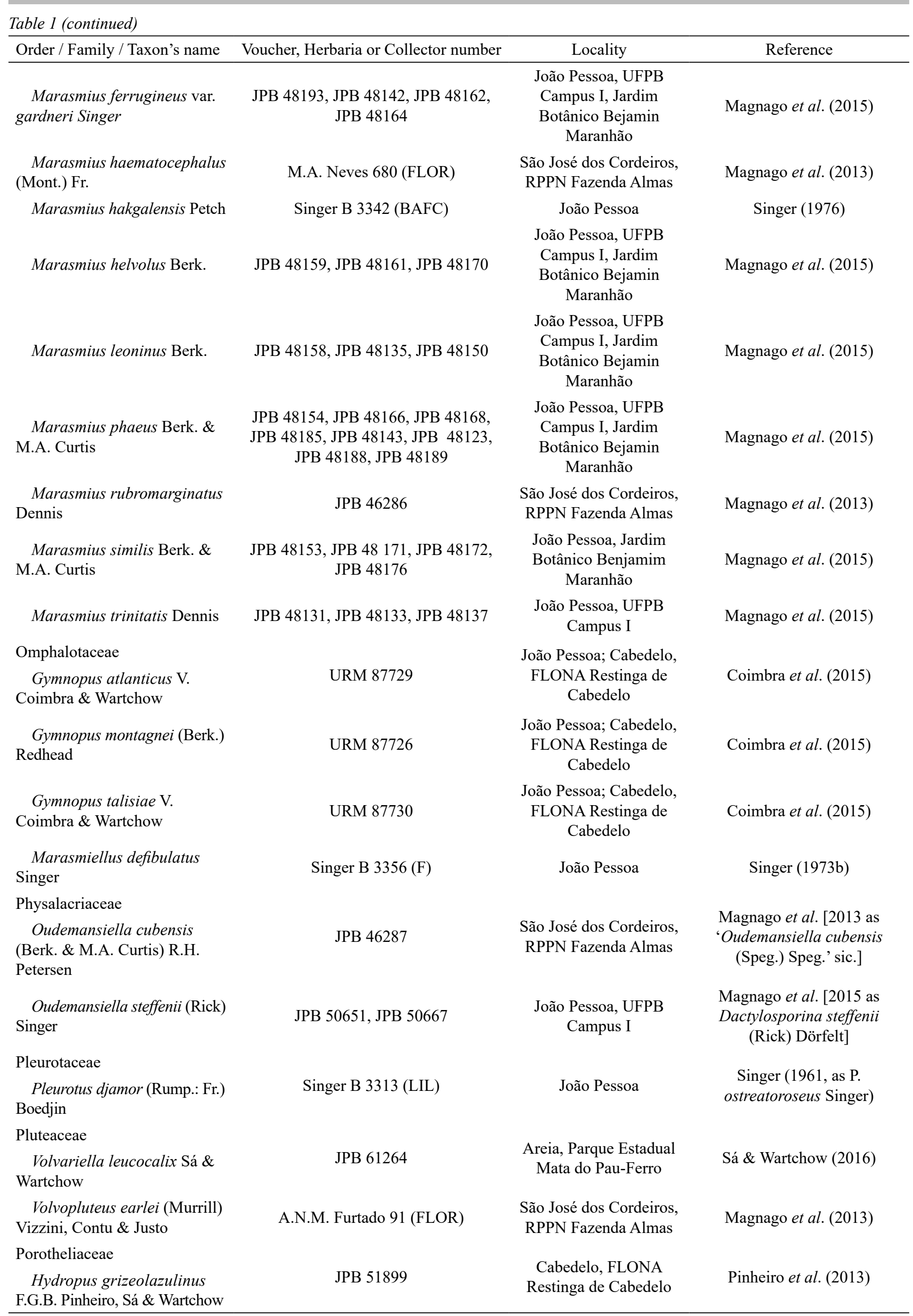


Table 1 (continued)

Order / Family / Taxon's name Voucher, Herbaria or Collector number

Psathyrellaceae

Locality

Reference

Coprinellus arenicola

JPB 56051

Wartchow \& A.R. Gomes

Coprinellus disseminatus

(Pers.) J.E. Lange

JPB 50668

Parasola leiocephala (P.D.

Orton) Redhead, Vilgalys \&

JPB 46290

Hopple

Psathyrella tuberculata (Pat.)

A.H. Sm.

JPB 46291

Strophariaceae

Agrocybe retigera (Speg.)

JPB 46293

Singer

Tricholomataceae

Leucopaxillus gracilimus

JPB 50662

Singer \& A.H. Sm.

\section{Agaricales}

Incertae sedis

Trogia cantharelloides (Mont.)

Singer

Singer B 3358 (LIL)

JPB 6891

Boletaceae

Boletellus lepidospora E.-J. Gilbert

Boletellus nordestinus A.C. Magnago

Boletellus pustulatus (Beeli)

E.-J. Gilbert

Chalciporus piperatus

(Bull.: Fr.) Batt.

Fistulinela ruschii A.C.

Magnago

Fistulinella violaceospora (G. Stev.) Pegler \& T.W.K Young

Tylopilus aquarius var. megistus Wartchow, Barbosa-Silva, B. Ortiz \& Ovrebo

Tylopilus nigripes Barbosa-

Silva \& Wartchow

Xerocomus amazonicus Singer

Boletinellaceae

Phlebopus beniensis

(Singer \& Digilio) Heinem. \& Rammeloo
FLOR 51603, FLOR 51604

JPB 6896, JPB 6893, JPB 6894, JPB 6595, JPB 6900, JPB 6897, JPB 6898, JPB 6899, JPB 6902, JPB 6901, JPB 6903, JPB 6904, JPB 6907, JPB 6906, JPB 6905, JPB 9277, JPB 6892, JPB

17503, JPB 17548, JPB 18626

JPB 6909, JPB 6908, JPB 6910, JPB 9420, JPB 9573, JPB 9276, JPB 8365, JPB 17522, JPB 17504, JPB 17551, JPB 17500, JPB 5914, JPB 18602, JPB 14734

JPB 6912, JPB 6911, JPB 6914, JPB 9429, JPB 9437, JPB 9438, JPB 6988, JPB 6990, JPB 17558, JPB 17550, JPB 17595

JPB 61780, JPB 61781, JPB 51100, UFRN-Fungos 2676, UFRN-Fungos 2493, URM 89205

JPB 63855

JPB 6973

JPB 6968, JPB 6969, JPB 6972, JPB 6986, JPB 9417, JPB 17563, JPB 17577 , JPB 18627

\section{ICN 192820}

João Pessoa

Cabedelo, FLONA

Restinga de Cabedelo

João Pessoa, Jardim

Botânico Benjamim

Maranhão

São José dos Cordeiros, RPPN Fazenda Almas

São José dos Cordeiros, RPPN Fazenda Almas

São José dos Cordeiros, RPPN Fazenda Almas

João Pessoa, UFPB

Campus I

Gomes \& Wartchow (2014)

Magnago et al. (2015)

Magnago et al. (2013)

Magnago et al. [2013 as 'Psathyrella tuberculata (Berk. \& Broome) P.D. Orton' sic.]

Magnago et al. (2013)

Magnago et al. (2015)

João Pessoa, UFPB

Campus I

Oliveira \& Sousa (1995)

Mamanguape, REBio Guaribas

Magnago et al. (2019)

João Pessoa, Campus I UFPB

Oliveira \& Sousa (1995)

João Pessoa, Campus I UFPB

João Pessoa, Campus I UFPB

Magnago et al. (2018)

João Pessoa, Campus I UFPB

Oliveira \& Sousa (2002)

Mamanguape, REBio Guaribas

Barbosa-Silva et al. (2017)

Mamanguape, REBio Guaribas

João Pessoa, Campus I UFPB

Barbosa-Silva et al. (2020)

Oliveira \& Sousa (1995)

João Pessoa, Campus I UFPB
Oliveira \& Sousa (1996) 


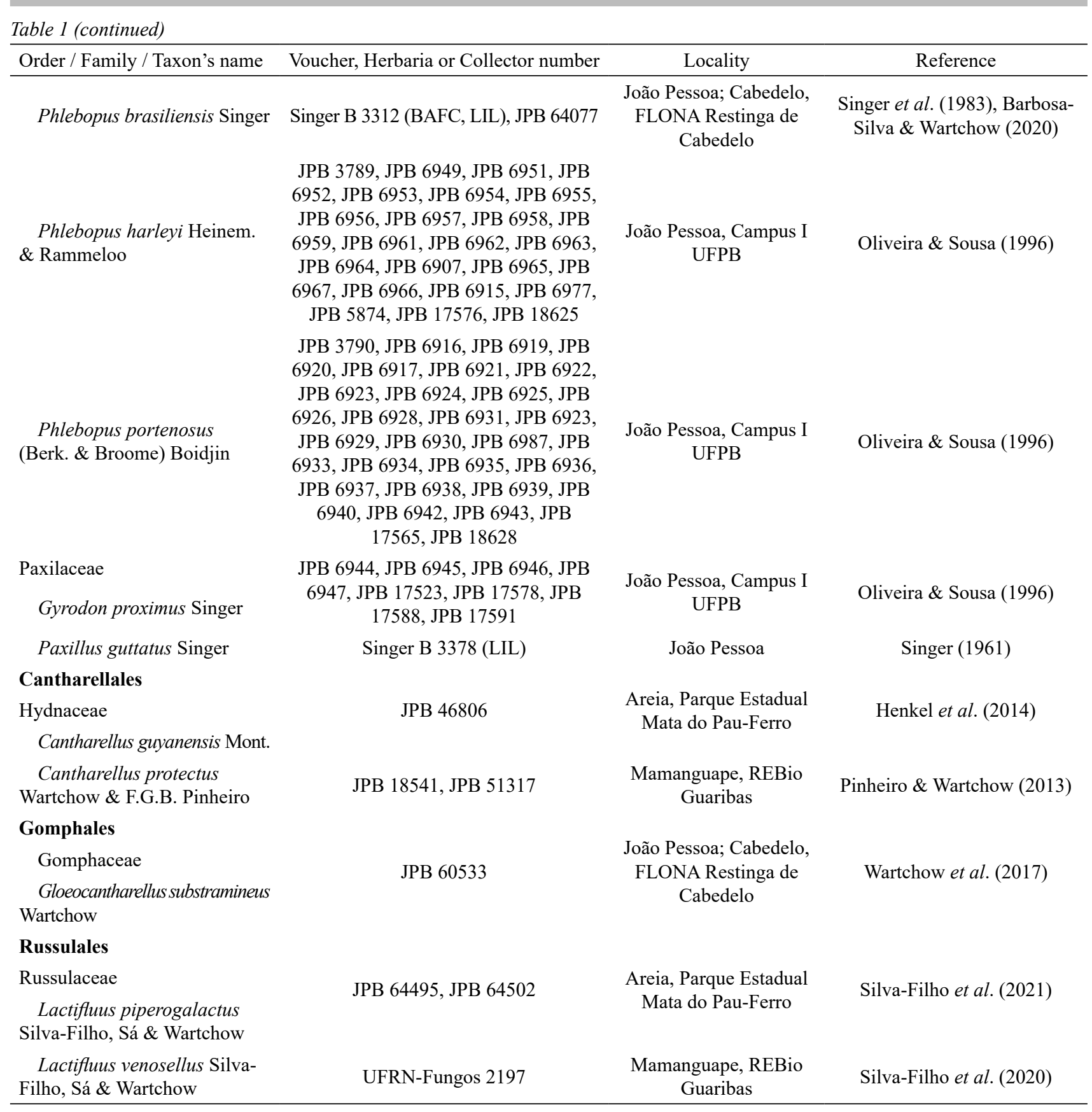

Cabedelo (FLONA Restinga de Cabedelo), João Pessoa (Jardim Botânico Benjamin Maranhão and UFPB Campus I), Mataraca (Millenium Inorganic Chemicals Minning a Crystal Company - now Tronox) and Mamanguape (REBio Guaribas) in the Atlantic Forest biome; and Areia (Parque Estadual Mata do Pau-Ferro), Juazeirinho (Fazenda Unha de Gato), and São José dos Cordeiros (RPPN Fazenda Almas) in the Caatinga biome (figure 1). Most of these specimens are deposited at JPB, but also in the herbaria of BAFC, F, FLOR, LIL, UFRN-Fungos and URM.

In João Pessoa were recorded at least two well delimited areas, UFPB Campus I and Jardim Botânico Benjamin Maranhão. All species published by Singer (1961, 1965, 1966, 1973a, 1973b, 1976, 1977) were delimited only with the city's name (table 1), and no exact locality was able to be informed. In Mataraca, it was recorded a mining currently named 'Tronox'.
Regarding to genera, Marasmius was the genus with the most species recorded for the state. We found 10 species cited or described (table 1). The genus comprises about 600 worldwide known species, most of them saprophytic (He et al. 2019, Wijayawardene et al. 2020), and recent studies referred 49 taxa, mostly from Atlantic Forest of Southeastern Brazil (e.g. Oliveira et al. 2014, 2020a, 2020b). The genus is considered a key component in the tropical mycobiota, due to the importance in decomposing leaf litter in forest by the networks of rhizomorphs that facilitate the trap litter (Lodge \& Cantrell 1995), and plays an important role in the nutrient cycle (Braga-Neto et al. 2008).

We also noted 15 species with putative Ectomycorrhizal (ECM) habit belonging to families Amanitaceae (Amanita), Boletaceae (Boletellus Murrill, Chalciporus Bataille, Fistulinella Henn. and Tylopilus P. Karst.), Hydnaceae (Cantharellus Juss.) and Russulaceae [Lactifluus (Pers.) Roussel] (Smith \& Read 2008). 
Some of them were found in the REBio Guaribas, an area with tableland vegetation with white sandy soil (Thomas \& Barbosa 2008). Interestedly, ECM macrofungi are much diverse in this kind of substrate (Roy et al. 2016) and are important to help their plant hosts to uptake nutrients from this poor-nutrient substrate (Smith \& Read 2008).

\section{General remarks and conclusion}

In Paraíba State, Magnago et al. (2013) cited 22 names of agaricoid species from the semiarid and Magnago et al. (2015) 18 species from the Atlantic Forest. However, some names published by them need to be re-analyzed for a more accurate identification. As an example, we can mention the material identified as Coprinus xerophilus Bogart by Magnago et al. (2013) which currently corresponds to C. calyptratus Peck (Gomes \& Wartchow 2018). Thus, most of the names reported may be a misapplication of European species names and need to be revised. For this reason, we have included all information as possible of the collection data for each name.

Taxonomic knowledge about agaricoid fungi in Brazil is still little understood, requiring more specific researches and focus in this group of macrofungi, and also among other diverse groups of the Kingdom Fungi.

In order to increase the knowledge about the high biodiversity of macrofungi belonging to this group in the Paraíba State, it is essential to have as a basis the research and revision of information on studies already carried out, making a survey of species already found and cataloged. We did not find studies published on ecological aspects of agaricoid fungi from Paraíba State. This is due to the lack of investment in research in the state, resulting in the scarcity of information of fungal species in the region.

\section{Acknowledgements}

We wish thank Conselho Nacional de Desenvolvimento Científico e Tecnológico (CNPq), by funding the projects Programa de Pesquisa em Biodiversidade (PPBio Proc. 60/2009), and Fungos agaricoides em áreas de Mata Atlântica e Caatinga no Estado da Paraíba (Edital Universal Proc. 420.448/2016-0), and by the concession of Produtividade em Pesquisa (Proc. 307922/2014-6 and Proc. 307947/2017-3), grant for FW and Iniciação Científica - IC (Proc. 307947/2017-3) for JCVA.

\section{Author Contributions}

Juliane C. Valões-Araújo: Substantial contribution in the concept and design of the study; Contribution to data collection; Contribution to data analysis and interpretation; Contribution to manuscript preparation.

Felipe Wartchow: Substantial contribution in the concept and design of the study; Contribution to critical revision. Contribution in supervising the lead author.

\section{Conflicts of interest}

There is no conflict of interest.

\section{Literature cited}

Barbosa-Silva, A. \& Wartchow, F. 2020. Considerations on the rare species Phlebopus brasiliensis (Fungi, Basidiomycota, Boletinellaceae) from Atlantic Forest of Northeast Brazil. Proceedings of the Biological Society of Washington 133: 109-119.

Barbosa-Silva, A., Ovrebo, C.L., Ortiz-Santana, B., Sá, M.C.A., Sulzbacher, M.A., Roy, M. \& Wartchow, F. 2017. Tylopilus aquarius, comb. et stat. nov., and its new variety from Brazil. Sydowia 69: 115-122.

Barbosa-Silva, A., Sulzbacher, M.A. \& Wartchow, F. 2020. Tylopilus nigripes sp. nov. (Boletaceae, Basidiomycota) from the Atlantic Forest of Brazil. Feddes Repertorium 131: 244-250.

Binder, M. \& Hibbett, D.S. 2006. Molecular systematic and biological diversification of Boletales. Mycologia 98: 971-981.

Braga-Neto, R., Luizão, R.C.C., Magnusson, W.E., Zuquim, G. \& Castilho, C.V. 2008. Leaf litter fungi in a Central Amazonian Forest: the influence of Rainfall, soil and topography on the distribution of fruiting bodies. Biodiversity and Conservation 17: 2701-2712.

Brandon, K., Fonseca, G.A.B., Rylands, A.B. \& da Silva, J.M.C. 2005. Conservação brasileira: desafios e oportunidades. Megadiversidade 1: 7-13.

Coimbra, V.R.M., Pinheiro, F.G.B., Wartchow, F. \& Gibertoni, T.B. 2015. Studies on Gymnopus sect. Impudicae (Omphalotaceae, Agaricales) from Northern Brazil: two new species and notes on G. montagnei. Mycological Progress 14: 110.

Crous, P.W., Gams, W., Stalpers, J.A., Robert, V. \& Stegehuis, G. 2004. MycoBank: an online initiative to launch mycology into $21^{\text {st }}$ century. Studies in Mycology 50: 19-22.

Dentinger, B.T.M., Gaya, E., O’Brien, H., Suz, L.M., Lachlan, R., Díaz-Valderrama, J.R., Koch, R.A. \& Aime, M.C. 2016. Tales from the crypt: genome mining from fungarium specierns improves resolution of the mushroom tree of life. Biological Journal of the Linnean Society 117: 11-32.

Francisco, P.R.M., Medeiros, R.M., Santos, D. \& Matos, R.M. 2015. Classificação climática de Köppen e Thornthwaite para o Estado da Paraíba. Revista Brasileira de Geografia Física 8: 1006-1016.

Funk, V.A., Sakai, A.K. \& Richardson, K. 2002 Biodiversity: the interface between systematics and conservation. Systematic biology 51: 235-237.

Gomes, A.R.P. \& Wartchow, F. 2014. Coprinellus arenicola, a new species from Paraíba, Brazil. Sydowia 66: 249-256.

Gomes, A.R.P. \& Wartchow, F. 2018. Notes on two coprinoid fungi (Basidiomycota, Agaricales) from the Brazilian semiarid region. Edinburgh Journal of Botany 75: 285-295.

Governo do Estado Paraíba. 2006. PERH-PB. Plano estadual de recursos hídricos: Resumo Executivo \& Atlas. Consórcio TC/BR Concremat, Brasilia. 
Hawksworth, D.L. 1991. The fungal dimension of biodiversity: magnitude, significance, and conservation. Mycological Research 95: 641-655.

He, M.Q., Zhao, R.L., Hyde, K.D., Begerow D., Kemler, M., Yurkov A., McKenzie E.H.C., Raspé, O., Kakishima M., Sánchez-Ramírez, S., Vellinga E.C., Halling, R.E., Papp. V., Zmitrovich, I.V., Buyck, B., Ertz, D., Wijayawardene, N.N., Cui, B.K., Schoutteten, N., Liu, X.Z., Li, T.H., Yao, Y.J., Zhu, X.Y., Liu, A.Q., Li, G.J., Zhang M.Z., Ling, Z.L., Cao, B., Antonín, V., Boekhout, T., da Silva, B.D.B., De Crop, E., Decock, C., Dima, B., Dutta, A.K., Fell, J.W., Geml, J., Ghobad-Nejhad, M., Giachini A.J., Gibertoni, T.B., Gorjón, S.P., Haelewaters, D., He, S.H., Hodkinson, B.P., Horak, E., Hoshino, T., Justo, A., Lim, Y.W., Menolli Jr., N., Mešić, A., Moncalvo, J.-M., Mueller, G.M., Nagy, L.G., Nilsson, R.H., Noordeloos, M., Nuytinck, J., Orihara, T., Ratchadawan, C., Rajchenberg, M., Silva-Filho, A.G.S., Sulzbacher, M.A., Tkalčec, Z., Valenzuela, R., Verbeken, A., Vizzini, A., Wartchow, F., Wei, T.Z., Weiß, M., Zhao, C.L. \& Kirk, P.M. 2019. Notes, outline and divergence times of Basidiomycota. Fungal Diversity 99: 105-367.

Henkel, T.W., Wilson, A.W., Aime, M.C., Dierks, J., Uehling, J.K., Roy, M., Schimann, H., Wartchow, F. \& Mueller, G.M. 2017. Cantharellaceae of Guyana II: New species of Craterellus, new South American distribution records for Cantharellus guyanensis and Craterellus excelsus, and a key to the Neotropical taxa. Mycologia 106: 307-322.

Horak, E. 1977. Entoloma in South America. I. Sydowia 30: 40-111.

Hosaka, K., Bates, S.T., Beever, R.E., Castellano, M.A., Colgan II, W., Domínguez, L.S., Nouhra, E.R., Geml, J., Giachini, A.J., Kenney, S.R., Simpson, N.B., Spatafora, J.W. \& Trappe, J. 2006. Molecular phylogenetics of the gomphoid-phalloid fungi with an establishment of the new subclass Phallomycetidae and two new orders. Mycologia 98: 949-959.

IBGE. 2004. Mapas de Cobertura Vegetal dos Biomas Brasileiros. Ministério do Meio Ambiente, Ministério do Planejamento, Orçamento e Gestão Instituto Brasileiro de Geografia e Estatística, Diretoria de Geociências Brasilia.

Kalichman, J., Kirk, P.M. \& Matheny, P.B. 2020. A compendium of generic names of agarics and Agaricales. Taxon 69: 425-447.

Lodge, D.J. \& Cantrell, S. 1995. Fungal communities in wet tropical forests: variation in time and space. Canadian Journal of Botany 73: S1391-S1398.

Lodge, D.J., Chapela, I., Samuels, G., Uecker, F.A., Desjardin, D., Horak, E., Miller Junior, O.K., Hennebert, G.L., Decock, C.A., Ammirati, J., Burdsall Junior, H.H., Kirk, P.M., Minter, D.W., Halling, R., Lassøe, T., Mueller, G.M., Huhnmdorf, S., Oberwinkler, F., Pegler, D.N., Spooner, B., Petersen, R.H., Rogers, J.D., Ryvarden, L., Watling, R., Turnbull, E. \& Whalley,A.J.S. 1995. A survey of patters of Diversity in non-lichenized fungi. Mitteilungen der Eidgenössischen Forschungsanstalt für Wald, Schnee und Landschaft 70: 157-173.
Magnago, A.C., Oliveira, J.J.S., Furtado, A.N.M., Urrea-Valencia, S. \& Neves, M.A. 2013. Mushroomscogumelos. In: Neves, M.A., Baseia, I.G., DrechslerSantos, E.R. \& Góes-Neto, A. Guide to the common fungi of the semiarid region of Brazil, TECC Editora, Florianópolis, pp. 24-49.

Magnago, A.C., Furtado, A.N.M., Urrea-Valencia, S., Freitas, A.F. \& Neves, M.A. 2015. New records of agaricoid fungi (Basidiomycota) from Paraíba, Brazil. Biotemas 28: 9-21.

Magnago, A.C., Neves, M.A. \& Silveira, R.M.B. 2017. Fistulinella ruschii sp. nov., and a new record of Fistulinella campinaranae var. scrobiculata for the Atlantic Forest, Brazil. Mycologia 109: 1003-1013.

Magnago, A.C., Neves, M.A. \& Silveira, R.M.B. 2019. Boletellus nordestinus (Boletaceae, Boletales), a new species from Northeastern Atlantic Forest, Brazil. Studies in Fungi 4: 47-53.

Matheny, P.B., Curtis, J.M., Hofstetter, V., Aime, M.C., Moncalvo, J.-M., Ge, Z.-W., Yang, Z.-L., Slot, J.G., Ammirati, J.F., Baroni, T.J., Bougher, N.L., Hughes, K.W., Lodge, D.J., Kerrigan, R.W., Seidl, M.T., Aanen, D.K., DeNitis, M., Daniele, G.M., Desjardin, D.E., Kropp, B.R., Norvell, L.L., Parker, A., Vellinga, E.C., Vilgalys, R. \& Hibbett, D.S. 2006. Major clades of Agaricales: a multilocus phylogenetic overview. Mycologia 98: 982-995.

Miller, S.L., Larsson, E., Larsson, K.-H., Verbeken, A. \& Nuytinck, J. 2006. Perspectives in the new Russulales. Mycologia 98: 960-970.

Moncalvo, J.-M., Nilsson, R.H., Koster, B., Dunham, S.M., Bernauer, T., Matheny, P.B., Porter, T.M., Margaritescu, S., Weiß, M., Garnica, S., Danell, E., Langer, G., Langer, E., Larsson, E., Larsson, K.-H. 2006. The cantharelloid clade: dealing with incongruent trees and phylogenetic reconstruction methods. Mycologia 98: 937-948.

Nascimento, C.C. \& Wartchow, F. 2018. Limacella brunneovenosa (Amanitaceae: Agaricomycetidae: Basidiomycota), a new species of Limacella sect. Amanitella from Brazilian Atlantic Forest. Current Research in Environmental \& Applied Mycology 8: 372-379.

Oliveira, I.C. \& Sousa, M.A. 1995. Boletales (Hymenomycetes) no Campus I da Universidade Federal da Paraíba, João Pessoa: I - Xerocomatae. Revista Nordestina de Biologia 10: 85-99.

Oliveira, I.C. \& Sousa, M.A. 1996 - Boletales (Hymenomycetes) no Campus I da Universidade Federal da Paraíba, João Pessoa: II - Gyrodontae. Revista Nordestina de Biologia 11: 97-117.

Oliveira, I.C. \& Sousa, M.A. 2002 - Boletales (Hymenomycetes) no Campus I da Universidade Federal da Paraíba, João Pessoa: III - Strobilomycetaceae. Revista Nordestina de Biologia 16: 43-53.

Oliveira, J.J.S., Sanchez-Ramirez, S. \& Capelari, M. 2014. Some new species and new varieties of Marasmius (Marasmiaceae, Basidiomycota) from Atlantic Rainforest areas of São Paulo state, Brazil. Mycological Progress 13: 923-949. 
Oliveira, J.J.S., Moncalvo, J.-M., Margaritescu, S. \& Capelari, M. 2020a. Phylogenetic and morphological analyses of species of Marasmius sect. Marasmius from the Atlantic Rainforest, Brazil. Plant Systematic and Evolution 306: 31.

Oliveira, J.J.S., Moncalvo, J.-M., Margaritescu, S. \& Capelari, M. 2020b. A morphological and phylogenetic evaluation of Marasmius sect. Globulares (GlobularesSicci complex) with nine new taxa from the Neotropical Atlantic Forest. Persoonia 44: 240-277.

Robert, V., Stegehuis, G. \& Stalpers, J.A. 2005. The MycoBank engine and related databases. Available at https://www.mycobank.org/ (access on 29-I-2021).

Sá, M.C.A. \& Wartchow, F. 2016. Volvariella leucocalix (Pluteaceae), a new species from Brazilian semiarid region. Mycosphere 7: 30-35.

Silva-Filho, A.G.S., Meiras-Ottoni, A. \& Wartchow, F. 2019. Hygrocybe aurantiomagnifica: a new species of section Firmae (Hygrophoraceae, Basidiomycota) from Brazil. Kew Bulletin 74: 63.

Silva-Filho, A.G.S., Sá, M.C.A., Komura, D.L., Moncalvo, J.-M., Margaritescu, S., Roy, M. \& Wartchow, F. 2020. Two novel species of Lactifluus subg. Pseudogymnocarpi (Russulaceae) from Brazil. Phytotaxa 436: 222-236.

Silva-Filho, A.G.S., Sá, M.C.A., Komura, D.L., Baseia, I.G., Marinho, P., Moncalvo, J.-M., Roy, M. \& Wartchow, F. 2021. Novelties in Lactifluus subg. Gymnocarpi (Russulales, Basidiomycota) from Brazilian tropical forests. Mycological Progress 20: 549-565.

Silva-Junior, F.C.S. \& Wartchow, F. 2015. Gymnopilus purpureograminicola (Strophariaceae, Agaricomycetidae), a new species from Paraíba, Brasil. Nova Hedwigia 101: 395-402.

Singer, R. 1961. Fungi of Northern Brazil. Publicação do Instituto de Micologia da Universidade do Recife 304: $1-26$.

Singer, R. 1965. Interesting and new Agaricales from Brazil. Atas do Instituto de Micologia da Universidade do Recife 2: 15-59.

Singer, R. 1966. Monographs of South American Basidiomycetes, especially those of the east slope of the Andes and Brazil. IX. Tricholoma in Brazil and Argentina. Darwiniana 14: 19-35.

Singer, R. 1973a. Diagnoses Fungorum Novorum Agaricalium III. Beihefte zur Sydowia 7: 1-106.

Singer, R. 1973b. The genera Marasmiellus, Crepidotus and Simocybe in the neotropics. Beihefte zur Nova Hedwigia 44: 1-517.

Singer, R. 1976. Marasmieae (Basidiomycetes Tricholomataceae). Flora Neotropica 17: 1-347.

Singer, R. 1977. Keys for identification of the species of Agaricales I. Sydowia 30: 192-279.

Singer, R. 1986. The Agaricales in Modern Taxonomy. $4^{\text {th }}$ ed., Koeltz Scientific Books, Stuttgart.

Singer, R., Araujo, I.J.A. \& Ivory, M.H. 1983. The ectotrophically mycorrhizal fungi of the neotropical lowlands, especially Central Amazonia. Beihefte zur Nova Hedwigia 77: 1-339.
Smith, S.E. \& Read, D.J. 2008. Mycorrhizal Symbiosis. $3^{\text {rd }}$ ed. Academic Press, New York.

Straatsma, G., Ayer, F. \& Egli, S. 2001. Species richness, abundance, and phenology of fungal fruit bodies over 21 years in a Swiss forest plot. Mycological Research 105: 515-523.

Sulzbacher, M.A., Orihara, T., Grebenc, T., Wartchow, F., Smith, M.E., Martín, M.P., Giachini, A.J. \& Baseia, I.G. 2020. Longistriata flava (Boletaceae, Basidiomycota) - a new monotypic sequestrate genus and species from Brazilian Atlantic Forest. MycoKeys 62: 53-73.

Thiers, B. 2020 [continuously updated] Index Herbariorum: A global directory of public herbaria and associated staff. New York Botanical Garden's Virtual Herbarium. Available at http://sweetgum.nybg.org/ih/ (access on 15 December 2020).

Thomas, W.W. \& Barbosa, M.R.V. 2008. Natural vegetation types in the Atlantic Coastal Forest of Hortheastern brazil. Memoirs of the New York Botanic Garden 100: 6-20.

Wartchow, F., Maia, L.C. \& Cavalcanti, M.A.Q. 2012. Studies on Amanita (Agaricomycetidae, Amanitaceae) in Brazil: two yellow gemmatoid taxa. Nova Hedwigia 96: 61-71.

Wartchow, F., Sulzbacher, M.A., Selosse, M.-A., Grebenc, T., Aime, M.C., Sá, M.C.A., Pinheiro, F.G.B., Baseia, I.G. \& Ovrebo, C.L. 2015. Sebacina aureomagnifica, a new heterobasidiomycete from the Atlantic Forest of Northeast Brazil. Mycological Progress 14: 109.

Wartchow, F., Sá, M.C.A. \& Coimbra, V.R.M. 2017. A new species of Gloeocantharellus from the Atlantic Forest of Paraíba, Brazil. Current Research in Environmental \& Applied Mycology 7: 183-186.

Wijayawardene, N.N., Hyde, K.D., Al-Ani, L.K.T., Tedersoo, L., Haelewaters, D., Rajeshkumar, K.C., Zhao, R.L., Aptroot, A., Leontyev, D.V., Saxena, R.K., Tokarev, Y.S., Dai, D.Q., Letcher, P.M., Stephenson, S.L., Ertz, D., Lumbsch, H.T., Kukwa, M., Issi, I.V., Madrid, H., Phillips, A.J.L., Selbmann, L., Pfliegler, W.P., Horváth, E., Bensch, K., Kirk, P.M., Kolaříková, K., Raja, H.A., Radek, R., Papp, V., Dima, V., Ma, J., Malosso, E., Takamatsu, S., Rambold, G., Gannibal, P.B., Triebel, D., Gautam, A.K., Avasthi, S., Suetrong, S., Timdal, E., Fryar, S.C., Delgado, G., Réblová, M., Doilom, M., Dolatabadi, S., Pawłowska, J.Z., Humber, R.A., Kodsueb, R., Sánchez-Castro, I., Goto, B.T., Silva, D.K.A., de Souza, F.A., Oehl, F., da Silva, G.A., Silva, I.R., Błaszkowski, J., Jobim, K., Maia, L.C., Barbosa, F.R., Fiuza, P.O., Divakar, P.K., Shenoy, B.D., Castañeda-Ruiz, R.F., Somrithipol, S., Lateef, A.A., Karunarathna, S.C., Tibpromma, S., Mortimer, P.E., Wanasinghe, D.N., Phookamsak, R., Xu, J., Wang, Y., Tian, F., Alvarado, P., Li, D.W., Kušan, I., Matočec, N., Mešic, A., Tkalčec, Z., Maharachchikumbura, S.S.N., Papizadeh, M., Heredia, G., Wartchow, F., Bakhshi, M., Boehm, E., Youssef, N., Hustad, V.P., Lawrey, J.D., Santiago, 
A.L.C.M.A., Bezerra, J.D.P., Souza-Motta, C.M., Firmino, A.L., Tian, Q., Houbraken, J., Hongsanan, S., Tanaka, K., Dissanayake, A.J., Monteiro, J.S., Grossart, H.P., Suija, A., Weerakoon, G., Etayo, J., Tsurykau, A., Vázquez, V., Mungai, P., Damm, U., Li, Q.R., Zhang, H., Boonmee, S., Lu, Y.Z., Becerra, A.G., Kendrick, B., Brearley, F.Q., Motiejūnaitė, J., Sharma, B., Khare, R., Gaikwad, S., Wijesundara, D.S.A., Tang, L.Z., He, M.Q., Flakus, A., Rodriguez-Flakus, P., Zhurbenko, M.P., McKenzie, E.H.C., Stadler, M., Bhat, D.J., Liu, J.K., Raza, M., Jeewon, R., Nassonova, E.S., Prieto, M., Jayalal, R.G.U., Erdoğdu, M., Yurkov, A., Schnittler,
M., Shchepin, O.N., Novozhilov, Y.K., Silva-Filho, A.G.S., Gentekaki, E., Liu, P., Cavender, J.C., Kang, Y., Mohammad, S., Zhang, L.F., Xu, R.F., Li, Y.M., Dayarathne, M.C., Ekanayaka, A.H., Wen, T.C., Deng, C.Y., Pereira, O.L., Navathe, S., Hawksworth, D.L., Fan, X.L., Dissanayake, L.S., Kuhnert, E., Grossart, H.P. \& Thines, M. 2020. Outline of Fungi and fungus-like taxa. Mycosphere 11: 1060-1456.

Received: 18.10 .2020

Accepted: 16.03 .2021

Associate Editor: Viviana Motato-Vásquez 